Pacific Journal of 


\section{COMBINATORIAL STRUCTURES IN LOOPS II. COMMUTATIVE INVERSE PROPERTY CYCLIC NEOFIELDS OF PRIME-POWER ORDER}

\section{E. C. Johnsen ANd Thomas Storer}

In this paper we construct a large family of commutative inverse property, cyclic (CIP) neofields of prime-power order. Our purpose in doing so is to produce a class of algebraic systems which shall be useful in certain combinatorial constructions. One of these constructions is that of power-residue difference sets in the additive loops of finite CIP neofields which is a natural generalization of the corresponding constructions in the additive groups of finite fields. Another is that of cyclic Steiner triple systems, i.e., Steiner triple systems with a cyclic group of automorphisms sharply transitive on elements, which we discuss in the last section of this paper.

CIP neofields may be thought of as a first generalization of finite fields in that they share all of the familiar properties of the fields with the possible exception of additive associativity. The present approach, accordingly, is to begin with a finite field and modify the additive structure thereon so as to preserve these properties. We show that the number of nonisomorphic CIP neofields of prime-power order $v=p^{\alpha}$ goes to infinity with $v$ and we exhibit proper (i.e., not the field) CIP neofields for every prime-power order $v=p^{\alpha} \geqq 11$ (every CIP neofield of order $v<11$ is a field). For $p=2$ the latter implies that there exists at least two nonisomorphic cyclic Steiner triple systems of order $2^{\alpha}-1 \geqq 15$. The constructions of powerresidue difference sets in finite CIP neofields appears in [5], the corresponding constructions in finite fields in [6], [9].

2. Preliminaries. A neofield of order $v$ is a triple $N_{v}=\langle N$, $+, \cdot\rangle$, where $N$ is a set of $v$ elements including 0 and 1 , and + and - are binary operations on $N$ such that $N(+)$ is a loop with identity element $0, N^{*}(\cdot)$ (where $N^{*}=N-\{0\}$ ) is a group with identity element 1 , and $\cdot$ is both left and right distributive over + . We also write $N_{v}$ for $N$ and $N_{v}^{*}$ for $N^{*}$. It is easily verified that $0 \cdot x=$ $0=x \cdot 0$ for every $x \in N_{v}$. The neofield $N_{v}$ is said to have the right inverse property (RIP) if for each $y \in N_{v}$ there is an element $z \in N_{v}$ such that $(x+y)+z=x$ for all $x \in N_{v}$, and to have the left inverse property (LIP) if for each $y \in N_{v}$ there is an element $w \in N_{v}$ such that $w+(y+x)=x$ for all $x \in N_{v}$. If $N_{v}$ has both the RIP and LIP then $N_{v}$ is called an inverse property (IP) neofield. It is readily verified that in an RIP or LIP neofield $N_{v}$ every $y \in N_{v}$ has a unique two-sided 
negative $-y \in N_{v}$. In fact, in the above definitions the elements $z$ and $w$ are this element $-y$. In an RIP or LIP neofield $N_{v}, x+(-1) x=$ $(1+(-1)) x=0=((-1)+1) x=(-1) x+x$, hence the negative of $x$ is $-x=(-1) x$ for every $x \in N_{v}$. We call a neofield $N_{v}$ commutative if $N_{v}(+)$ is commutative. The following result is probably known.

\section{Lemma 2.1. An IP neofield is commutative.}

Proof. Let $N_{v}$ be an IP neofield and let $x, y \in N_{v}$ with $x+y=z$. By the RIP we have $x=(x+y)+(-y)=z+(-y)$, by the LIP this becomes $(-z)+x=(-z)+(z+(-y))=-y$, and by the RIP again we obtain $-z=((-z)+x)+(-x)=(-y)+(-x)$ or $(-1) z=(-1) y+$ $(-1) x=(-1)(y+x)$. Since $-1 \in N_{v}^{*}$ we obtain $z=y+x$. Hence $x+y=y+x$, and we see that $N_{v}$ is commutative.

We call a neofield $N_{v}$ cyclic when $\boldsymbol{N}_{v}^{*}(\cdot)$ is cyclic. Let $\boldsymbol{N}_{v}$ be a cyclic neofield. A presentation of $N_{v}$ based on the set $N$ is the expression of $N$ in terms of a multiplicative generator $a, N=\{0,1$, $\left.a, a^{2}, \cdots, a^{v-2}\right\}$ where $a^{n-1}=1$, together with a function $T: N \rightarrow N$, called the presentation function, which is related to the addition in $N_{v}$ by $1+x \equiv T(x)$ for all $x \in N$. In a cyclic neofield the element 1 has a unique two-sided negative -1 where $-1=1$ if $v$ is even and $-1=a^{(v-1) / 2}$ if $v$ is odd ([7], p. 49, Theorem II2). Using the presentation of a cyclic neofield $\boldsymbol{N}_{v}$ we can construct the addition table $\hat{N}_{v}$ for $N_{v}(+)$. We choose the natural order $0,1, a, a^{2}, \cdots, a^{v-2}$ for the first row and first column of $\hat{N}_{v}$. Then the second row of $\hat{N}_{v}$ consists of the elements $T(0)=1, T(1), T(\alpha), T\left(a^{2}\right), \cdots, T\left(a^{v-2}\right)$ in this order. By the distributive laws we have

$$
a^{r}+a^{s}=a^{r}\left(1+a^{s-r}\right)=a^{r} T\left(a^{s-r}\right) ; 0 \leqq r, s \leqq v-2,
$$

hence the table $\hat{N}_{v}$ is completely determined by its first and second rows. A cyclic neofield $N_{v}$ is thus completely determined by its presentation; henceforth, we shall give a presentation of $\boldsymbol{N}_{v}$ in terms of the first two rows of $\hat{N}_{v}$. Note, however, that an abstract cyclic neofield of order $v$ may have more than one presentation. For example, the unique finite field of order 7 has the presentations

\begin{tabular}{|lllllll}
\hline 0 & 1 & $a$ & $a^{2}$ & $a^{3}$ & $a^{4}$ & $a^{5}$ \\
\hline 1 & $a^{2}$ & $a^{4}$ & $a$ & 0 & $a^{5}$ & $a^{3}$ \\
\hline
\end{tabular} and \begin{tabular}{|lllllll|}
0 & 1 & $a$ & $a^{2}$ & $a^{3}$ & $a^{4}$ & $a^{5}$ \\
\hline 1 & $a^{4}$ & $a^{3}$ & $a$ & 0 & $a^{5}$ & $a^{2}$ \\
\hline
\end{tabular} .

Different presentations of a cyclic neofield $N_{v}$, of course, correspond to different definitions of addition on the set $N$. Finally, we call a cyclic IP neofield a CIP neofield. 
3. Construction of a family of CIP neofields. Let $\boldsymbol{F}_{v}=\langle F$, $+, \cdot\rangle$ be the finite field of order $v=p^{\alpha} \geqq 11, p$ prime, $\alpha \geqq 1$ integral, with presentation given by $F=\left\{0,1, a, a^{2}, \cdots, a^{v-2}\right\}$ and the presentation function $T$ for which $T(x)=1+x$ for all $x \in F$. We define the functions $T^{\prime}$ and $T_{0}$ on $F$ as follows:

$$
\begin{array}{r}
T^{\prime}(x) \equiv(-1)+x, \quad x \in F, \\
T_{0}(x) \equiv\left\{\begin{array}{l}
T(x), x=0,-1 \\
\frac{x}{T(x)}, \text { otherwise },
\end{array}\right.
\end{array}
$$

and define a new addition $\oplus$ on $F$ according to

$$
x \oplus y \equiv\left\{\begin{array}{l}
y ; y \in F, x=0 \\
x T_{0}\left(x^{-1} y\right) ; x, y \in F, x \neq 0 .
\end{array}\right.
$$

Also, we define

$$
T_{0}^{\prime}(x) \equiv(-1) \oplus x, x \in F .
$$

We note that $\boldsymbol{F}_{v}^{(0)}=\langle F, \oplus, \cdot\rangle$ is also the field of order $v=p^{\alpha}$ which, as the image of the mapping $0 \rightarrow 0, x \rightarrow x^{-1}$ for all $x \neq 0$ in $F$, is an isomorph but not an automorph of $\boldsymbol{F}_{v}(+, \cdot)$. We let the corresponding presentation of $\boldsymbol{F}^{(0)}$ be given by $F=\left\{0,1, a, a^{2}, \cdots, a^{v-2}\right\}$ and the presentation function $T_{0}$. We shall be concerned with compositions of the functions $T, T^{\prime}$, and $T_{0}$ on the set $F$. We need the following two results for the neofield construction which is to follow.

\section{Lemma 3.1. For all $x \in F,\left(T^{\prime \prime} T_{0}\right)^{3}(x)=x$.}

Proof. We easily verify that $T^{\prime} T_{0}(0)=0$ and $T^{\prime} T_{0}(-1)=-1$, hence the lemma holds for $x=0,-1$. We now take $x \neq 0,-1$. Then a straightforward computation yields $T^{\prime} T_{0}(x)=-(1+x)^{-1}$. Since $-(1+x)^{-1} \neq 0,-1$, a second application of $T^{\prime} T_{0}$ yields $\left(T^{\prime} T_{0}\right)^{2}(x)=$ $-(1+x) x^{-1}$. Finally, since $-(1+x) x^{-1} \neq 0,-1$, a third application of $T^{\prime} T_{0}$ yields $\left(T^{\prime} T_{0}\right)^{3}(x)=x$; hence the lemma.

We now determine those $x \in F$ for which $T^{\prime} T_{0}(x)=x$.

Lemma 3.2. We have $\left(T^{\prime} T_{0}^{\prime}\right)(x)=x$ (i.e., $\left.T(x)=T_{0}(x)\right)$ in the set $\boldsymbol{F}_{v}, v=p^{\alpha}$, precisely when

(1) $x=0,-1$. (This includes $1=-1$ when $p=2$.)

(2) $p=3$ and $x=1$. (Here $T(1)=-1$.)

(3) $p^{\alpha} \equiv 1(\bmod 3)$ and $x$ is a primitive cube root of unity in $\boldsymbol{F}_{v}$. (Here $T(x)=-x^{2}$ is a primitive sixth root of unity in $\boldsymbol{F}_{v}$ when $p \neq 2$.) 
Proof. We already have (1) from the proof of the previous lemma. When $1 \neq-1 v$ is odd and $T^{\prime} T_{0}(1)=1$ if and only if $p=$ $\operatorname{char}\left(\boldsymbol{F}_{v}\right)=3$ and $T(1)=-1$. When $x \neq 0,1,-1$ we have $T^{\prime} T_{0}(x)=$ $x$ if and only if $1+x+x^{2}=0$. Here $x$ is a primitive cube root of unity in $\boldsymbol{F}_{v}$, and $x^{3}=1$ implies that $3 \mid v-1$ or $p^{\alpha} \equiv 1(\bmod 3)$. Also, $T(x)=1+x=-x^{2}$ satisfies $(1+x)^{2}-(1+x)+1=0$ and is thus a primitive sixth root of unity in $\boldsymbol{F}_{v}$ when $p \neq 2$.

CoRollary 3.3. Let $S=\left\{x \in F \mid\left(T^{\prime} T_{0}\right)(x) \neq x\right\}$. Then $S$ is partitioned into triples $\left\{y, T^{\prime} T_{0}(y),\left(T^{\prime} T_{0}\right)^{2}(y)\right\}$, whence $|S| \equiv 0(\bmod 3)$.

Proof. The elements $y, T^{\prime} T_{0}(y)$, and $\left(T^{\prime} T_{0}\right)^{2}(y)$ are distinct except when $y$ is one of the elements given in Lemma 3.2 and thus satisfies $T^{\prime} T_{0}(y)=y$. Hence $S$ is partitioned into triples and $|S| \equiv 0(\bmod 3)$.

We now change viewpoint and assume that $N_{v}=\langle F, \boxplus, \cdot\rangle$ is a cyclic neofield of order $v=p^{\alpha}$ with presentation given by $F$ and the presentation function $T_{*}$ satisfying

(i) $T_{*} \not \equiv T$ and $T_{*} \not \equiv T_{0}$ on $F$,

(ii) for each $x \in F$, either $T_{*}(x)=T(x)$ or $T_{*}(x)=T_{0}(x)$. We inquire as to what other conditions $T_{*}$ must satisfy on $F$. Immediate restrictions are obtained in the following result.

Lemma 3.4. The function $T_{*}$ is bijective on $F$, and for all $x, y \in$ $F$ we must have

(1) $T_{*}(x) \neq x$,

(2) $x T_{*}(y) \neq T_{*}(x y)$ for $x \neq 1$.

Furthermore, $N_{v}$ is commutative if and only if

(3) $\quad x T_{*}\left(x^{-1}\right)=T_{*}(x)$ for all $x \neq 0$ in $F$.

Proof. That $T_{*}$ is bijective and satisfies (1) and (2) is obvious. In $N_{v}$ we automatically have $x \boxplus 0=0 \boxplus x$ for all $x \in F$. Suppose $x, y \in F$ where $x \neq 0 \neq y$. Then

$$
x \boxplus y=x\left(1 \boxplus x^{-1} y\right)=y\left(x y^{-1}\right) T_{*}\left(\left(x y^{-1}\right)^{-1}\right)
$$

and $y \boxplus x=y\left(1 \boxplus x y^{-1}\right)=y T_{*}\left(x y^{-1}\right)$. Hence $x \boxplus y=y \boxplus x$ if and only if $\left(x y^{-1}\right) T_{*}\left(\left(x y^{-1}\right)^{-1}\right)=T_{*}\left(x y^{-1}\right)$. Let $z=x y^{-1}$. As $x$ and $y$ run over $N_{v}^{*}$ so does $z$, and as $z$ runs over $N_{v}^{*}$ all pairs $x, y \in N_{v}^{*}$ are obtained. Hence, $N_{v}$ is commutative if and only if $z T_{*}\left(z^{-1}\right)=T_{*}(z)$ for all $z \neq$ 0 in $F$, which is (3).

For $y \in S=\left\{x \in F \mid T^{\prime} T_{0}(x) \neq x\right\}$ we define the orbit of $y$ to be the set $\theta(y) \equiv\left\{y, T^{\prime} T_{0}(y),\left(T^{\prime} T_{0}\right)^{2}(y)\right\}$. A simple computation shows that $\theta(y)=\left\{y,(-1) / T(y),(-1) / T_{0}(y)\right\}$. We now show that $T_{*}$ is identically $T$ or $T_{0}$ on the orbits in $S$. 
LEMmA 3.5. If $T_{*}$ agrees with $T$ (or $T_{0}$ ) at $y \in S$, then $T_{*}$ agrees with $T\left(\right.$ or $\left.T_{0}\right)$ on $\theta(y)$.

Proof. We first note that for $y \in S$, the two sets

$$
T(\theta(y))=\left\{T(y), T\left(T^{\prime} T_{0}\right)(y), T\left(T^{\prime} T_{0}\right)^{2}(y)\right\}
$$

and

$$
T_{0}(\theta(y))=\left\{T_{0}(y), T_{0}\left(T^{\prime} T_{0}\right)(y), T_{0}\left(T^{\prime} T_{0}\right)^{2}(y)\right\}
$$

are equal, since

$$
T_{0}(y)=T\left(T^{\prime \prime} T_{0}\right)(y), T_{0}\left(T^{\prime \prime} T_{0}\right)(y)=T\left(T^{\prime} T_{0}\right)^{2}(y),
$$

and $T_{0}\left(T^{\prime} T_{0}\right)^{2}(y)=T\left(T^{\prime} T_{0}\right)^{3}(y)=T(y)$. Suppose $T_{*}(y)=T(y)$. If $T_{*}\left(T^{\prime} T_{0}\right)^{2}(y)=T_{0}\left(T^{\prime} T_{0}\right)^{2}(y)$ then $T_{*}\left(T^{\prime} T_{0}\right)^{2}(y)=T(y)$, contrary to the fact that $T_{*}$ is injective. Hence $T_{*}\left(T^{\prime} T_{0}\right)^{2}(y)=T\left(T^{\prime} T_{0}\right)^{2}(y)$. Further, if $T_{*}\left(T^{\prime \prime} T_{0}\right)(y)=T_{0}\left(T^{\prime} T_{0}\right)(y)$ then $T_{*}\left(T^{\prime} T_{0}\right)(y)=T\left(T^{\prime} T_{0}\right)^{2}(y)$, again contrary to $T_{*}$ being injective. Hence $T_{*}\left(T^{\prime} T_{0}\right)(y)=T\left(T^{\prime} T_{0}\right)(y)$. Thus, if $T_{*}$ agrees with $T$ at $y \in S$ then $T_{*}$ agrees with $T$ on $\theta(y)$. Similarly, if $T$ agrees with $T_{0}$ at $y \in S$ then $T_{*}$ agrees with $T_{0}$ on $\theta(y)$.

When $N_{v}$ is commutative the condition $x T\left(x^{-1}\right)=T(x)$ (or $x T_{0}\left(x^{-1}\right)=$ $T_{0}(x)$ ) effects a further agreement of $T_{*}$ and $T$ (or $T_{0}$ ) on the orbits in $S$.

Lemma 3.6. Suppose $N_{v}$ is commutative. If $T_{*}$ agrees with $T$ (or $\left.T_{0}\right)$ at $y \in S$, then $T_{*}$ agrees with $T\left(\right.$ or $\left.T_{0}\right)$ on $\theta(y) \cup \theta\left(y^{-1}\right)$. Thus, the orbits in $S$ are paired except when $1 \in S$. In the latter case $\theta(1)$ is paired with itself.

Proof. Suppose $T_{*}(y)=T(y)$. Then, by Lemma 3.5, $T_{*}$ agrees with $T$ on $\theta(y)$. Since $N_{v}$ is commutative we have, by Lemma 3.4(3), that $y T_{*}\left(y^{-1}\right)=T_{*}(y)$, whence $y T_{*}\left(y^{-1}\right)=T(y)=y T\left(y^{-1}\right)$ or $T_{*}\left(y^{-1}\right)=$ $T\left(y^{-1}\right)$. Again, by Lemma 3.5, $T_{*}$ agrees with $T$ on $\theta\left(y^{-1}\right)$. Hence $T_{*}$ agrees with $T$ on $\theta(y) \cup \theta\left(y^{-1}\right)$. Now, $\theta(y)=\theta\left(y^{-1}\right)$ if and only if one of $y=y^{-1}, y=T^{\prime} T_{0}\left(y^{-1}\right)=-1 / T\left(y^{-1}\right)$, or

$$
y=\left(T^{\prime} T_{0}\right)^{2}\left(y^{-1}\right)=(-1) / T_{0}\left(y^{-1}\right)
$$

holds.

Case 1. $y=y^{-1}$. Here $y^{2}=1$, hence $y=1$ since $-1 \notin S$, whence $\theta(y)=\theta(1)$.

Case 2. $y=(-1) / T\left(y^{-1}\right)$. Here $y=-1 /\left(1+y^{-1}\right)=(-y) /(y+1)$ or $y=-2$, whence $\theta(y)=\left\{-2,1,-2^{-1}\right\}=\theta(1)$. 
Case 3. $y=(-1) / T_{0}\left(y^{-1}\right)$. Here $y=(-y)\left(1+y^{-1}\right)=-y-1$ or $y=-2^{-1}$, whence $\theta(y)=\left\{-2^{-1},-2,1\right\}=\theta(1)$.

Clearly, when $1 \in S, \theta(1)$ is paired with itself. A similar argument goes through when $T_{*}(y)=T_{0}(y)$.

We now show that if $N_{v}$ is commutative then it inherits the IP from the field. Since $N_{v}$ is cyclic -1 is also the negative of 1 in $N_{v}(\boxplus)$; whence, $-y=(-1) y$ is the negative of $y$ in $N_{v}(\boxplus)$ for every $y \in N_{v}$.

Lemma 3.7. If $\boldsymbol{N}_{v}$ is commutative, then $\boldsymbol{N}_{v}$ is an IP neofield.

Proof. Since $\boldsymbol{N}_{v}$ is assumed to be commutative, we only need to prove that $N_{v}$ has the LIP. Now $(-y) \boxplus(y \boxplus 0)=0$ for all $y \in F$ and $(-0) \boxplus(0 \boxplus x)=x$ for all $x \in F$, hence we are left with proving $(-y) \boxplus(y \boxplus x)=x$ for all $x \neq 0 \neq y$. Now, $(-y) \boxplus(y \boxplus x)=x$ if and only if $(-1) \boxplus\left(1 \boxplus x y^{-1}\right)=x y^{-1}$ or $T_{*}^{\prime} T_{*}\left(x y^{-1}\right)=x y^{-1}$, where $T_{*}^{\prime}$ is defined by $T_{*}^{\prime}(w)=(-1) \boxplus w$ for all $w \in F$. Let $z=x y^{-1}$. As $x$ and $y$ run over $N_{v}^{*}$ so does $z$, and as $z$ runs over $N_{v}^{*}$ every pair $x, y \in N^{*}$ is obtained. Hence $N_{v}$ has the IP if and only if $T_{*}^{\prime} T_{*}(z)=z$ for all $z \neq 0$ in $N_{v}$. If $T^{\prime} T_{0}(z)=z$ then $T_{*}(z)=T(z)=$ $T_{0}(z)$, and regardless of whether $T_{*}^{\prime}$ agrees with $T^{\prime}$ or $T_{0}^{\prime}$ on $T_{*}(z)$ we have $T_{*}^{\prime} T_{*}(z)=z$. Otherwise, $T^{\prime} T_{0}(z) \neq z$ and $z$ has an orbit $\theta(z)=$ $\left\{z,(-1) / T(z),(-1) / T_{0}(z)\right\}$. For $\operatorname{such} z,(-1) \boxplus(1 \boxplus z)=(1 \boxplus z) \boxplus(-1)=$ $(1 \boxplus z)(1 \boxplus(-1) /(1 \boxplus z)) \quad$ or $\quad T_{*}^{\prime \prime} T_{*}(z)=T_{*}(z) T_{*}\left((-1) / T_{*}(z)\right)$, and, by Lemma 3.5 and the commutativity and IP of $\boldsymbol{F}_{v}(+)$ and $\boldsymbol{F}_{v}^{(0)}(\oplus)$, we have

$$
T_{*}^{\prime} T_{*}(z)=\left\{\begin{array}{lll}
T(z) T((-1) / T(z))=T^{\prime} T(z)=z & \text { if } & T_{*}(z)=T(z) \\
T_{0}(z) T_{0}\left((-1) T_{0}(z)\right)=T_{0}^{\prime} T_{0}(z)=z & \text { if } \quad T_{*}(z)=T_{0}(z)
\end{array}\right.
$$

Hence $T_{*}^{\prime} T_{*}(z)=z$ for all $z \neq 0$ in $N_{v}$, whence $N_{v}$ has the IP, as was to be shown.

We now have enough information on the neofield $N_{v}=\langle F, \boxplus, \cdot\rangle$ obtained from $\boldsymbol{F}_{v}$ and $\boldsymbol{F}_{v}^{(0)}$ according to (i) and (ii) to give a construction of CIP neofields for every prime-power order $v=p^{\alpha} \geqq 11$.

ThEOREM 3.8. Let $\boldsymbol{F}_{v}=\langle F,+, \cdot\rangle$ and $\boldsymbol{F}_{v}^{(0)}=\langle F, \oplus, \cdot\rangle, F=\{0$, $\left.1, a, a^{2}, \cdots, a^{v-2}\right\}, a^{v-1}=1$, be two copies of the finite field of order $v \geqq 11$ with presentation functions $T$ and $T_{0}$, respectively, where $T_{0}$ is related to $T$ by

$$
T_{0}(x)=\left\{\begin{array}{l}
T(x), x=0,-1 \\
\frac{x}{T(x)}, x \neq 0,-1
\end{array}\right.
$$

Let $T_{*}$ be any mapping on $F$ satisfying 
(a) $T_{*} \not \equiv T$ and $T_{*} \not \equiv T_{0}$ on $F$,

(b) for each $x \in F$, either $T_{*}(x)=T(x)$ or $T_{*}(x)=$ $T_{0}(x)$,

(c) if $T_{*}$ agrees with $T$ (or $T_{0}$ ) at $x \in S$, then $T_{*}$ agrees with $T\left(\right.$ or $\left.T_{0}\right)$ on $\theta(x) \cup \theta\left(x^{-1}\right)$.

${ }_{\text {Then }} T_{*}$ is the presentation function for a CIP neofield $N_{v}=\langle F, \boxplus, \cdot\rangle$.

Proof. Let $N_{v}(\boxplus)$ be the groupoid on $F$ defined by

$$
x \boxplus y \equiv \begin{cases}y, & x=0 \\ x T_{*}\left(x^{-1} y\right), & x \neq 0\end{cases}
$$

for all $x, y \in F$. Now, by (3.7), $0 \boxplus y=y$ and $x \boxplus 0=x T_{*}(0)=x T(0)=$ $x T_{0}(0)=x \cdot 1=x$ for all $x, y \in N_{v}$; hence 0 is the identity element in $N_{v}(\boxplus)$ and 0 commutes with every element in $\boldsymbol{N}_{v}$. Let $x \neq 0$ in $\boldsymbol{N}_{v}$ and suppose that $T_{*}(x)=T(x)$. Then, by $(3.6)(\mathrm{c}), T_{*}\left(x^{-1}\right)=T\left(x^{-1}\right)$, hence $x T_{*}\left(x^{-1}\right)=x T\left(x^{-1}\right)=T(x)=T_{*}(x)$. If $T_{*}(x)=T_{0}(x)$ we again obtain $x T_{*}\left(x^{-1}\right)=T_{*}(x)$. By the proof of (3) of Lemma 3.4, this implies that $N_{v}(\boxplus)$ is commutative. Now, $0 \boxplus x=b$ has the unique solution $x=b$ for any $b \in N_{v}$. By (3.7), $a \boxplus x=b$ for $a, b \in N_{v}, a \neq 0$, has a unique solution $x \in N_{v}$ if and only if $T_{*}\left(\alpha^{-1} x\right)=\alpha^{-1} b$ has, that is, if and only if $T_{*}$ is bijective on $N_{v}$. Suppose for $w, z \in N_{v}, w \neq z$, we have $T_{*}(w)=T_{*}(z)$. Now $T_{*}$ agrees with one of $T$ and $T_{0}$ at $w$ and, since both $T$ and $T_{0}$ are bijective, $T_{*}$ must agree with the other at $z$. We may assume that $T_{*}(w)=T(w)$ and $T_{*}(z)=T_{0}(z)$. Then $T_{0}(z)=T(w)$ or $T^{\prime} T_{0}(z)=w \neq z$, hence $z$ has an orbit $\theta(z)$ and $w \in \theta(z)$. By (3.6) (c) this means that $T_{*}$ agrees with $T_{0}$ at $w$, whence $T_{0}(w)=T_{*}(w)=$ $T_{*}(z)=T_{0}(z)$, which contradicts the fact that $T_{0}$ is bijective. Hence, $T_{*}$ is bijective on $N_{v}$ and, by the commutativity of $N_{v}(\boxplus), x \boxplus a=$ $a \boxplus a=b$ always has a unique solution $x \in N_{v}$ for every choice of elements $a, b \in N_{v}$. Hence $N_{v}(\boxplus)$ is a commutative loop. Now, for any $w \neq 0, x, y \in N_{v}$ we have, by (3.7), that $0 \cdot(x \boxplus y)=0=0 \cdot x \boxplus$ $0 \cdot y$ and

$$
w \cdot(x \boxplus y)= \begin{cases}w \cdot y=w \cdot 0 \boxplus w \cdot y=w \cdot x \boxplus w \cdot y, & x=0 \\ w \cdot x T_{*}\left(x^{-1} y\right)=w x T_{*}\left((w x)^{-1}(w y)\right)=w x \boxplus w y, & x \neq 0,\end{cases}
$$

hence, since $N_{v}(\cdot)$ is commutative, $\boxplus$ is both left and right distributive over $\cdot$. Thus $N_{v}(\boxplus, \cdot)$ is a cyclic commutative neofield. By (3.6) (a) and (b), $N_{v}$ satisfies the implicit conditions of Lemma 3.7, hence $N_{v}=\langle F, \boxplus, \cdot\rangle$ is a CIP neofield with presentation function $T_{*}$.

For $\boldsymbol{F}_{v}$ and $\boldsymbol{F}_{v}^{(0)}$ of order $v=p^{\alpha} \leqq 9$ we have $|S| / 3 \leqq 2$, and so condition (3.6) (a) cannot be satisfied. For $v=11$ and 13 we have $|S| / 3=3$ and $\theta(1)$ is paired with itself. Here, fixing $T_{*}=T$ (or $T_{0}$ ) 
on $S-\theta(1)$ and $T_{*}=T_{0}$ (or $T$ ) on $\theta(1)$ yields what we shall call a special CIP neofield. We remark that special CIP neofields exist for every order $v=p^{\alpha} \geqq 11$ where $p \neq 2,3$. The construction produces nonspecial CIP neofields for every order $v=p^{\alpha} \geqq 16$. At this point we do not know whether this construction produces neofields which are not isomorphs of the corresponding field. This question is taken up in the next section.

4. Proper CIP neofields. A neofield is called proper if it is not a field. It is natural to inquire as to which of the CIP neofields constructed by Theorem 3.8 are proper and how many nonisomorphic proper CIP neofields are obtained. So far we do not have the complete answer to these questions; however, we can obtain some information of value. Let $\phi$ denote the Euler phi-function. We need the following preliminary result.

Lemma 4.1. A cyclic neofield $N_{v}=\langle N,+, \cdot\rangle, N=\left\{0,1, a, a^{2}, \cdots\right.$, $\left.a^{v-2}\right\}$, of order $v>1$ has at most $\varphi(v-1)$ presentations based on the set $N$.

Proof. Let $T_{0}$ be the presentation function for $N_{v}^{(0)}=\langle N, \oplus, \cdot\rangle$, where $N_{v}^{(0)}$ is isomorphic to $N_{v}$. Let $\Psi$ denote the isomorphism from $N_{v}$ onto $N_{v}^{(0)}$. Then $\Psi$ induces an isomorphism from $N_{v}^{*}(\cdot)$ onto $N_{v}^{(0) *}(\cdot)$. In terms of the generator $a$ of $N_{v}^{*}(\cdot)$ we have $\Psi: a \rightarrow a^{r}$ where $a^{r}$ is a generator of $N_{v}^{(0) *}(\cdot)$. Since $\left|N_{v}^{(0)^{*}}(\cdot)\right|=v-1$ we must have ged $(r$, $v-1)=1$. Since $\Psi$ is completely determined by its effect on a multiplicative generator, the number of different presentations of $N_{v}$ on the set $N$ is at most the number of different integers $r, 1 \leqq r \leqq$ $v-1$ such that $\operatorname{gcd}(r, v-1)=1$, which is $\varphi(v-1)$.

THEOREM 4.2. The number of nonisomorphic CIP neofields of order $v=p^{\alpha}$ constructed by Theorem 3.8 goes to infinity with $v$.

Proof. In the construction of Theorem 3.8, let $u$ denote the number of elements $x$ such that $T^{\prime} T_{0}(x) \neq x$ and $x \notin \theta(1)$ if $\theta(1)$ exists. Then $u / 6$ is the number of orbit pairs $\theta(x) \cup \theta\left(x^{-1}\right)$ on which a choice of either $T$ or $T_{0}$ can be made. If $\theta(1)$ exists then the total number of neofield presentations constructed is $2^{(u / 6)+1}-2$ and if $\theta(1)$ does not exist, this number is $2^{u / 6}-2$. Now, the value of $u$ is $3^{\alpha}-3=$ $v-3$ if $p=3,2^{\alpha}-4=v-4$ if $p=2$ and $p^{\alpha} \equiv 1(\bmod 3), p^{\alpha}-7=$ $v-7$ if $p \neq 2$ and $p^{\alpha} \equiv 1(\bmod 3), 2^{\alpha}-2=v-2$ if $p=2$ and $p^{\alpha} \equiv$ $2(\bmod 3)$, and $p^{\alpha}-5=v-5$ if $p \neq 2$ and $p^{\alpha} \equiv 2(\bmod 3)$. Since $\theta(1)$ exists only for $p \neq 2,3$, the resulting number of neofield presentations is, respectively, $2^{(v-3) / 6}-2,2^{(v-4) / 6}-2,2^{(v-1) / 6}-2,2^{(v-2) / 6}-2$, and $2^{(v+1) / 6-2}$. 
Now, by Lemma 4.1, a given neofield of order $v$ can occur among these presentations at most $\varphi(v-1)$ times, hence the construction yields at least

$$
\frac{2^{(v-r) / 6}-2}{\varphi(v-1)}>\frac{2^{(v-r) / 6}-2}{v-1}
$$

nonisomorphic neofields of order $v$, where $r=-1,1,2,3$, or 4 . In any of these cases we clearly have

$$
\lim _{v \rightarrow \infty} \frac{2^{(v-r) / 6}-2}{v-1}=\infty,
$$

hence the theorem.

In the case of a field $\boldsymbol{F}_{v}$ of order $v=p^{\alpha}$ the number of different presentations given by the various isomorphisms $\Psi_{r}: a \rightarrow a^{r}$, gcd ( $r$, $v-1)=1$, is $\alpha^{-1} \varphi\left(p^{\alpha}-1\right)$ since the mappings $\Psi_{p^{i}}: a \rightarrow a^{p^{i}}, i=1,2,3, \cdots$, $\alpha$, are all automorphisms and automorphisms preserve presentations. The number of presentations of neofields of order $v=p^{\alpha}$ constructed by Theorem 3.8 is larger than $\alpha^{-1} \varphi\left(p^{\alpha}-1\right)-2$ for all $p^{\alpha} \geqq 11$ except 11,13, and 17 and for these orders the theorem constructs proper neofields by inspection. Hence, for all orders $v \geqq 11$, proper CIP neofields are constructed by Theorem 3.8. In the following theorems we give actual constructions of proper CIP neofields for each order $v=p^{\alpha} \geqq 11$, divided into the three cases where $p>7$, $p=3,5,7$, and $p=2$. The three analyses are rather distinct; each is based on particular properties of the case involved.

THeOREM 4.3. Let $v=p^{\alpha} \geqq 11$ where $p>7$. Then $2 \notin \theta(1)$ and any neofield $N_{v}$ of order $v$ constructed by Theorem 3.8 with $T_{*}=T$ on $\theta(1)$ and $T_{*}=T_{0}$ on $\theta(2)$ has the property that

$$
1 \boxplus(1 \boxplus(1 \boxplus 1)) \neq(1 \boxplus 1) \boxplus(1 \boxplus 1)
$$

and is, hence, not the field of order $v$.

Proof. We note that $\boldsymbol{F}_{p} \subseteq \boldsymbol{F}_{p^{\alpha}}=\boldsymbol{F}_{v}$ and that for $x \in \boldsymbol{F}_{v}, x \in \boldsymbol{F}_{p}$ if and only if $\theta(x) \subseteq \boldsymbol{F}_{p}$. Since $p \neq 2,3, \theta(1)$ exists and $\theta(1)=\left\{1,-2^{-1}\right.$, $-2\}$; hence $2 \in \theta(1)$ iff $5=0$ or $4=0$, i.e., $p=2$ or $p=5$. Thus, choosing $T_{*}=T$ on $\theta(1)$ and $T_{*}=T_{0}$ on $\theta(2)$,

$$
1 \boxplus(1 \boxplus(1 \boxplus 1))=T_{*} T_{*} T_{*}(1)=T_{*} T_{0} T(1)=T_{*}\left(\frac{2}{3}\right)
$$

and

$(1 \boxplus 1) \boxplus(1 \boxplus 1)=(1 \boxplus 1)(1 \boxplus 1)=T_{*}(1) T_{*}(1)=T(1) T(1)=4$. 
If $T_{*}(2 / 3)=T(2 / 3)=5 / 3$ then $T_{*}(2 / 3)=4$ implies that $7=0$, and if $T_{*}(2 / 3)=T_{0}(2 / 3)=2 / 5$ then $T_{*}(2 / 3)=4$ implies that $2=0$ or $3=0$, all of which are contradictions. Hence $T_{*}(2 / 3) \neq 4$, which is (4.1).

TheOREM 4.4. Let $v=p^{\alpha}>11$ where $p=3,5$, or 7. Let $x \in \boldsymbol{F}_{v}$ where $x \notin \boldsymbol{F}_{p}$ and $x \neq-1 \pm \sqrt{-1}, x \neq 2^{-1}(-1 \pm \sqrt{5}), x \neq 2^{-1}(-3 \pm$ $\sqrt{5}), x \neq 2^{-1}(-3 \pm \sqrt{-3})$, in case any of these elements exist in $\boldsymbol{F}_{v}$ and are not in $\boldsymbol{F}_{p}$. Then $T(x) \notin \theta(x) \cup \theta\left(x^{-1}\right)$, and any neofield $\boldsymbol{N}_{v}$ of order $v$ constructed by Theorem 3.8 with $T_{*}=T$ on $\theta(1) \cup \theta(x) \cup \theta\left(x^{-1}\right)$ and $T_{*}=T_{0}$ on $\theta(T(x)) \cup \theta\left((T(x))^{-1}\right)$ has the property that

$$
1 \boxplus(1 \boxplus x) \neq(1 \boxplus 1) \boxplus x
$$

and is, hence, not the field of order $v$.

Proof. We first note that the element $x$ must be different from at most $p+8$ elements of $\boldsymbol{F}_{v}$. Since the minimal values of $3^{\alpha}, 5^{\beta}$, and $7^{r}$ are 27, 25, and 49 and $p+8 \leqq 15$, such an element $x$ exists. Since $T(x) \notin \boldsymbol{F}_{p}$ we have $T(x) \notin \theta(1)$. Furthermore, $T(x) \in \theta(x) \cup \theta\left(x^{-1}\right)$ implies that either $x \in \boldsymbol{F}_{p}$ or $x$ is one of the first six forbidden values. Thus, choosing $T_{*}=T$ on $\theta(1) \cup \theta(x) \cup \theta\left(x^{-1}\right)$ and $T_{*}=T_{0}$ on $\theta(T(x)) \cup$ $\theta\left((T(x))^{-1}\right)$,

$$
1 \boxplus(1 \boxplus x)=T_{*} T_{*}(x)=T_{0} T(x)=(x+1)(x+2)^{-1}
$$

and

$$
\begin{aligned}
(1 \boxplus 1) \boxplus x & =x \boxplus(1 \boxplus 1)=x\left(1 \boxplus\left(x^{-1} \boxplus x^{-1}\right)\right) \\
& =x T_{*}\left(x^{-1} T(1)\right)=x T_{*}\left(2 x^{-1}\right) .
\end{aligned}
$$

If $T_{*}\left(2 x^{-1}\right)=T\left(2 x^{-1}\right)=1+2 x^{-1}$ then $x T_{*}\left(2 x^{-1}\right)=(x+1)(x+2)^{-1}$ implies that $x^{2}+3 x+3=0$ or $x$ is one of the last two forbidden values, and if $T_{*}\left(2 x^{-1}\right)=T_{0}\left(2 x^{-1}\right)=2(x+2)^{-1}$ then $x T_{*}\left(2 x^{-1}\right)=(x+1)(x+2)^{-1}$ implies that $x=1$, both of which are contradictions. Hence $(x+$ $1)(x+2)^{-1} \neq x T_{*}\left(2 x^{-1}\right)$, which is $(4.2)$.

THeOREM 4.5. Let $v=2^{\alpha}>11$ and let $x$ be any element in $\boldsymbol{F}_{v}$ which is not in the largest of the subfields $\boldsymbol{F}_{2}, \boldsymbol{F}_{4}$, or $\boldsymbol{F}_{8}$ contained in $\boldsymbol{F}_{v}$. Then $x^{2} \notin \theta(x) \cup \theta\left(x^{-1}\right)$ and any neofield $\boldsymbol{N}_{v}$ of order $v$ constructed by Theorem 3.8 with $T_{*}=T$ on $\theta(x) \cup \theta\left(x^{-1}\right)$ and $T_{*}=T_{0}$ on $\theta\left(x^{2}\right) \cup$ $\theta\left(x^{-2}\right)$ has the property that

$$
(x \boxplus 1) \boxplus x^{2} \neq x \boxplus\left(1 \boxplus x^{2}\right)
$$

and is, hence, not the field of order $v$.

Proof. Since $x$ is not in any subfield $\boldsymbol{F}_{2}, \boldsymbol{F}_{4}$, or $\boldsymbol{F}_{8}$ of $\boldsymbol{F}_{v}, x$ satisfies 
no cubic or lower degree equation over $\boldsymbol{F}_{2}$. If $x^{2} \in \theta(x) \cup \theta\left(x^{-1}\right)=\{x$, $\left.-(1+x)^{-1},-(1+x) x^{-1}, x^{-1},-x\left(1+x^{-1}\right),-(1+x)\right\}$, this condition is clearly violated. Thus, choosing $T_{*}=T$ on $\theta(x) \cup \theta\left(x^{-1}\right)$ and $T_{*}=T_{0}$ on $\theta\left(x^{2}\right) \cup \theta\left(x^{-2}\right)$,

$$
\begin{aligned}
(x \boxplus 1) \boxplus x^{2} & =x^{2} \boxplus(1 \boxplus x)=x^{2} T_{*}\left(x^{-2} T_{*}(x)\right) \\
& =x^{2} T_{*}\left(x^{-2} T(x)\right)=x^{2} T_{*}\left(x^{-1}+x^{-2}\right)
\end{aligned}
$$

and

$x \boxplus\left(1 \boxplus x^{2}\right)=x T_{*}\left(x^{-1} T_{*}\left(x^{2}\right)\right)=x T_{*}\left(x^{-1} T_{0}\left(x^{2}\right)\right)=x T_{*}\left(x\left(1+x^{2}\right)^{-1}\right)$.

If $x^{2} T_{*}\left(x^{-1}+x^{-2}\right)=x^{2} T\left(x^{-1}+x^{-2}\right)=x^{2}+x+1$ and $x T_{*}\left(x\left(1+x^{2}\right)^{-1}\right)=$ $x T\left(x\left(1+x^{2}\right)^{-1}\right)=x+x^{2}\left(1+x^{2}\right)^{-1}$ then $x^{2} T_{*}\left(x^{-1}+x^{-2}\right)=x T_{*}\left(x\left(1+x^{2}\right)^{-1}\right)$ implies that $x^{2}+x+1=0$, a contradiction. If

$$
x^{2} T_{*}\left(x^{-1}+x^{-2}\right)=x^{2} T\left(x^{-1}+x^{-2}\right)=x^{2}+x+1
$$

and $x T_{*}\left(x\left(1+x^{2}\right)^{-1}\right)=x T_{0}\left(x\left(1+x^{2}\right)^{-1}\right)=x^{2}\left(1+x+x^{2}\right)^{-1}$ then

$$
x^{2} T_{*}\left(x^{-1}+x^{-2}\right)=x T_{*}\left(x\left(1+x^{2}\right)^{-1}\right)
$$

implies that $x^{2}+1=0$, another contradiction. If $x^{2} T_{*}\left(x^{-1}+x^{-2}\right)=$ $x^{2} T_{0}\left(x^{-1}+x^{-2}\right)=\left(x^{2}+x^{3}\right)\left(1+x+x^{2}\right)^{-1}$ and $x T_{*}\left(x\left(1+x^{2}\right)^{-1}\right)=x T(x(1+$ $\left.\left.x^{2}\right)^{-1}\right)=x+x^{2}\left(1+x^{2}\right)^{-1}$ then $x^{2} T_{*}\left(x^{-1}+x^{-2}\right)=x T_{*}\left(x\left(1+x^{2}\right)^{-1}\right)$ implies that $x^{3}+x+1=0$, also a contradiction. Finally, if $x^{2} T_{*}\left(x^{-1}+x^{-2}\right)=$ $x^{2} T_{0}\left(x^{-1}+x^{-2}\right)=\left(x^{2}+x^{3}\right)\left(1+x+x^{2}\right)^{-1}$ and $x T_{*}\left(x\left(1+x^{2}\right)^{-1}\right)=x T_{0}(x(1+$ $\left.\left.x^{2}\right)^{-1}\right)=x^{2}\left(1+x+x^{2}\right)^{-1}$ then $x^{2} T_{*}\left(x^{-1}+x^{-2}\right)=x T_{*}\left(x\left(1+x^{2}\right)^{-1}\right)$ implies that $x=0$, again a contradiction. Hence $x^{2} T_{*}\left(x^{-1}+x^{-2}\right) \neq x T_{*}(x(1+$ $\left.x^{2}\right)^{-1}$ ) which establishes (4.3).

It is natural to inquire as to the orders for which CIP neofields exist. The order need not be a prime-power as the following presentation for the lone CIP neofield of order 14 shows:

\begin{tabular}{|c||cccccccccccccc|}
\hline$x$ & 0 & 1 & $a$ & $a^{2}$ & $a^{3}$ & $a^{4}$ & $a^{5}$ & $a^{6}$ & $a^{7}$ & $a^{8}$ & $a^{9}$ & $a^{10}$ & $a^{11}$ & $a^{12}$ \\
\hline$T_{*}(x)$ & 1 & 0 & $a^{4}$ & $a^{7}$ & $a^{12}$ & $a$ & $a^{11}$ & $a^{8}$ & $a^{2}$ & $a^{6}$ & $a^{10}$ & $a^{9}$ & $a^{5}$ & $a^{3}$ \\
\hline
\end{tabular}

Recently, John R. Doner has obtained CIP neofields for all orders $v \geqq 2$ satisfying $v \neq \equiv 0,6,12,15,18,21(\bmod 24)$ and $v \neq 10$ and has shown that no CIP neofields exist for these forbidden orders. Hughes [4] had earlier shown that the orders $v \equiv 0,6,12,18(\bmod 24)$ were forbidden, and the authors, among perhaps others, had earlier observed that order $v=10$ is also forbidden.

5. Cyclic Steiner triple systems. A Steiner triple system of order $n, S(n)$, is an arrangement of a set of $n$ elements into triples 
such that every pair of elements occur together in precisely one triple. A necessary and sufficient condition that an $S(n)$ exist is that $n \equiv$ $1,3(\bmod 6)$. An $S(n)$ is called cyclic if it has a cyclic group of automorphisms which is sharply transitive on the elements. For an excellent historical discussion and introduction to the literature on Steiner triple systems in general and cyclic Steiner triple systems in particular, the reader is referred to the first section of Doyen [3]. Here we note that a cyclic Steiner triple system $S(n)$ is known to exist for all orders $n \equiv 1,3(\bmod 6)$ except $n=9$ [8]. Now, a CIP neofield $N_{v}=\left\{0,1, a, a^{2}, \cdots, a^{v-2}\right\}$ of order $v=2^{\alpha}$ has the property that $x+x=0$ or $-x=x$ for all $x \in N_{v}$, and so if $x, y, z \in N_{v}$ satisfy $x+y=z$ then also $y+x=z, x+z=z+x=y$, and $y+z=z+$ $y=x$. This means that $N_{v}(+)$ is a totally symmetric loop. The set of elements $N_{v}^{*}=N_{v}-\{0\}$ formed into the triples $\{x, y, z\}$ where $x+y=z$ thus yields a Steiner triple system $S(n)$ of order $n=$ $v-1$ [2]. Furthermore, the right regular representation of $\boldsymbol{N}_{v}^{*}(\cdot)$ is a cyclic group of automorphisms of $N_{v}(+)$, hence also of $S(n)$, which is sharply transitive on the elements of $S(n)$. Hence, a CIP neofield of order $v=2^{\alpha} \geqq 4$ naturally yields a cyclic Steiner triple system of order $n=v-1$. Now, CIP neofields of order $v=2^{\alpha}$ with nonisomorphic additive loops yield nonisomorphic cyclic Steiner triple systems of order $n=2^{\alpha}-1$, and by Theorem 4.5 there exists both the field of order $v$ and a proper CIP neofield of order $v$ for every order $v=2^{\alpha} \geqq 16$. Hence, we obtain the following result, which is a more specific version of a theorem of Assmus and Mattson [1].

THEOREM 5.1. There exists at least two nonisomorphic cyclic Steiner triple systems for each order $n=2^{\alpha}-1 \geqq 15$.

Although the number of nonisomorphic CIP neofields of order $v=2^{\alpha}$ goes to infinity with $v$, we cannot immediately conclude from this that the number of nonisomorphic cyclic Steiner triple systems of order $v-1$ does the same, since we must ascertain the number of nonisomorphic additive loops among the nonisomorphic CIP neofields of order $v$. By further investigation, however, the authors have determined that this number does go to infinity with $v$. This will be presented in a subsequent paper.

\section{REFERENCES}

1. E. F. Assmus, Jr. and H. F. Mattson, On the number of inequivalent Steiner triple systems, J. Combinatorial Theory, 1 (1966), 301-305; Errata, 2 (1967), 394.

2. R. H. Bruck, What is a loop?, in Studies in Modern Algebra (M. A. A. Studies in Mathematics, 2), Prentice-Hall, Inc., Englewood Cliffs, N. J., (1963), 59-99.

3. Jean Doyen, Sur la croissance du nombre de systèmes triples de Steiner non isomorphes, J. Combinatorial Theory, 8 (1970), 424-441. 
4. D. R. Hughes, Planar division neo-rings, Trans. Amer. Math. Soc., 80 (1955), 502527.

5. E. C. Johnsen and Thomas Storer, Combinatorial structures in loops III. Difference sets in special cyclic neofields, J. Number Theory, to appear.

6. Emma Lehmer, On residue difference sets, Canad. J. Math., 5 (1953), 425-432.

7. Lowell J. Paige, Neofields, Duke Math. J., 16 (1949), 39-60.

8. Rose Peltesohn, Eine Lösung der beiden Heffterschen Differenzenprobleme, Compositio Math., 6 (1939), 251-257.

9. Thomas Storer, Cyclotomy and Difference Sets, Markham Publishing Co., Chicago, 1967.

Received December 12, 1972. The first author was supported in part by Air Force Office of Scientific Research Grants AFOSR 698-67 and 72-2163. The second author was supported in part by an NSF Research Grant.

University of California, Santa Barbara

AND

UNIVERSity of Michigan, ANN ARBOR 



\section{PACIFIC JOURNAL OF MATHEMATICS}

EDITORS

RICHARD ARENS (Managing Editor)

University of California

Los Angeles, California 90024

R. A. Beaumont

University of Washington

Seattle, Washington 98105

J. DugundJI

Department of Mathematics

University of Southern California

Los Angeles, California 90007

D. Gilbarg and J. Milgram

Stanford University

Stanford, California 94305

\section{ASSOCIATE EDITORS}

E. F. BECKENBACH

B. H. NeumanN

F. WOLF

K. YOSHIDA

\section{SUPPORTING INSTITUTIONS}

UNIVERSITY OF BRITISH COLUMBIA CALIFORNIA INSTITUTE OF TECHNOLOGY

UNIVERSITY OF CALIFORNIA

MONTANA STATE UNIVERSITY

UNIVERSITY OF NEVADA

NEW MEXICO STATE UNIVERSITY

OREGON STATE UNIVERSITY

UNIVERSITY OF OREGON

OSAKA UNIVERSITY

\author{
UNIVERSITY OF SOUTHERN CALIFORNIA \\ STANFORD UNIVERSITY \\ UNIVERSITY OF TOKYO \\ UNIVERSITY OF UTAH \\ WASHINGTON STATE UNIVERSITY \\ UNIVERSITY OF WASHINGTON \\ $\stackrel{*}{*} \stackrel{*}{*}{ }^{*}{ }^{*}{ }^{2}$ AMERICAN MATHEMATICAL SOCIETY \\ NAVAL WEAPONS CENTER
}




\section{Pacific Journal of Mathematics}

\section{Vol. 52, No. $1 \quad$ January, 1974}

David R. Adams, On the exceptional sets for spaces of potentials ............ 1

Philip Bacon, Axioms for the Čech cohomology of paracompacta ............ 7

Selwyn Ross Caradus, Perturbation theory for generalized Fredholm operators ..... 11

Kuang-Ho Chen, Phragmén-Lindelöf type theorems for a system of nonhomogeneous equations ............................ 17

Frederick Knowles Dashiell, Jr., Isomorphism problems for the Baire classes .......

M. G. Deshpande and V. K. Deshpande, Rings whose proper homomorphic images are right subdirectly irreducible . . . . . . . . . . . . . . . . . . . . . . . . .

Mary Rodriguez Embry, Self adjoint strictly cyclic operator algebras .............

Paul Erdős, On the distribution of numbers of the form $\sigma(n) / n$ and on some related

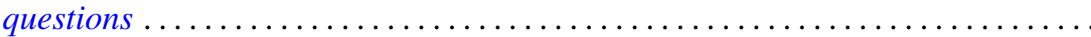

Richard Joseph Fleming and James E. Jamison, Hermitian and adjoint abelian

operators on certain Banach spaces ............................

Stanley P. Gudder and L. Haskins, The center of a poset .................. 85

Richard Howard Herman, Automorphism groups of operator algebras . . . ........

Worthen N. Hunsacker and Somashekhar Amrith Naimpally, Local compactness of families of continuous point-compact relations ....................

Donald Gordon James, On the normal subgroups of integral orthogonal groups ....

Eugene Carlyle Johnsen and Thomas Frederick Storer, Combinatorial structures in

loops. II. Commutative inverse property cyclic neofields of prime-power

order.

Ka-Sing Lau, Extreme operators on Choquet simplexes . . . . . . . . . . . . . . 129

Philip A. Leonard and Kenneth S. Williams, The septic character of 2, 3, 5 and $7 \ldots 143$

Dennis McGavran and Jingyal Pak, On the Nielsen number of a fiber map ........ 149

Stuart Edward Mills, Normed Köthe spaces as intermediate spaces of $L_{1}$ and



Philip Olin, Free products and elementary equivalence. .

Louis Jackson Ratliff, Jr., Locally quasi-unmixed Noetherian rings and ideals of the principal class.

Seiya Sasao, Homotopy types of spherical fibre spaces over spheres ...

Helga Schirmer, Fixed point sets of polyhedra ...

Kevin James Sharpe, Compatible topologies and continuous irreducible

representations.

Frank Siwiec, On defining a space by a weak base . . . . . . . . . . . . . . . 233

James McLean Sloss, Global reflection for a class of simple closed curves ....... 247

M. V. Subba Rao, On two congruences for primality . .

Raymond D. Terry, Oscillatory properties of a delay differential equation of even

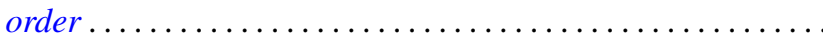

Joseph Dinneen Ward, Chebyshev centers in spaces of continuous functions . .

Robert Breckenridge Warfield, Jr., The uniqueness of elongations of Abelian

groups...

V. M. Warfield, Existence and adjoint theorems for linear stochastic differential

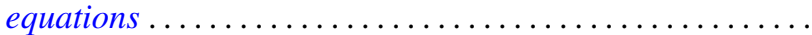

